\title{
Hunger can be taught: Hunger Recognition regulates eating and improves energy balance
}

This article was published in the following Dove Press journal:

International Journal of General Medicine

15 June 2013

Number of times this article has been viewed

\section{Mario Ciampolini' \\ H David Lovell-Smith ${ }^{2}$ \\ Timothy Kenealy ${ }^{3}$ \\ Riccardo Bianchi ${ }^{4}$}

'Unit of Preventive Gastroenterology, Department of Pediatrics, Università di Firenze, Florence, Italy;

2Department of General Practice, University of Otago, Christchurch, New Zealand; ${ }^{3}$ Department of General Practice and Primary Health Care, University of Auckland, Auckland, New Zealand; ${ }^{4}$ Department of Physiology and Pharmacology, State University of New York Downstate Medical Center, Brooklyn, NY, USA

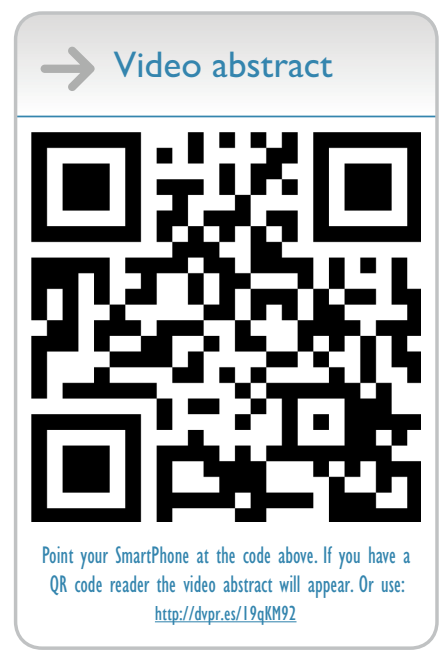

Correspondence: Mario Ciampolini Unit of Preventive Gastroenterology, Department of Pediatrics, Università di Firenze, Florence 50 132, Italy Tel +39055 215744

Emailmlciampolini@fastwebnet.it

\begin{abstract}
A set of spontaneous hunger sensations, Initial Hunger (IH), has been associated with low blood glucose concentration (BG). These sensations may arise pre-meal or can be elicited by delaying a meal. With self-measurement of BG, subjects can be trained to formally identify and remember these sensations (Hunger Recognition). Subjects can then be trained to ensure that $\mathrm{IH}$ is present pre-meal for most meals and that their pre-meal BG is therefore low consistently (IH Meal Pattern). IH includes the epigastric Empty Hollow Sensation (the most frequent and recognizable) as well as less specific sensations such as fatigue or lightheadedness which is termed inanition. This report reviews the method for identifying IH and the effect of the IH Meal Pattern on energy balance. In adults, the IH Meal Pattern has been shown to significantly decrease energy intake by one-third, decrease preprandial BG, reduce glycosylated hemoglobin, and reduce insulin resistance and weight in those who are insulin resistant or overweight. Young children as well as adults can be trained in Hunger Recognition, giving them an elegant method for achieving energy balance without the stress of restraint-type dieting. The implications of improving insulin sensitivity through improved energy balance are as wide as improving immune activity.
\end{abstract}

Keywords: energy intake, hunger, energy balance, food intake regulation, prevention, insulin resistance, obesity, diabetes, inflammation, risks

\section{Introduction}

This review outlines the authors' perspective on the current status and promising avenues for future research and clinical application of the recognition of hunger validated by pre-meal low blood glucose concentration (BG). The authors' work so far on low BG-associated hunger, the effect of such recognition on food intake regulation, and its clinical implications as a non-restraint dietary method in managing insulin resistance and obesity is reviewed.

\section{The theory}

Hypothesis: Food intake regulation improves when BG-validated hunger is recognized.

Prediction: Recognition of BG-validated hunger should lead to improved intake regulation and homeostatic energy balance as evidenced by improved insulin resistance and loss of weight in the overweight (OW).

\section{Research strategies}

The authors' investigations have taken the following course: 
1. Subjective assessment of hunger in infants by trained carers (MC).

2. Correlation of identified hunger with low BG concentration in infants (MC).

3. Self-identification of hunger sensations in adults (DLS, TK).

4. Correlation of identified hunger with low BG concentration in adults (MC, RB, DLS).

5. Identification of a meal pattern in which hunger and low BG is present pre-meal for most meals (the Initial Hunger [IH] Meal Pattern) (MC, RB, DLS).

6. Correlation of the IH Meal Pattern with loss of weight in the OW and with improved insulin sensitivity (MC, RB, DLS).

\section{Background}

The effects of positive energy balance are well known and underlie the great scourges of Western industrialized nations including obesity, diabetes, and heart disease. More than 1.1 billion adults worldwide are OW, and 312 million of them are obese ${ }^{1}$ obesity has been shown prospectively to be associated with dietary and lifestyle factors. ${ }^{2}$ Dieters' inability to lose weight long term by restraining food intake (dietary restraint) is well known. ${ }^{3}$ During dietary restraint-induced weight loss, appetite-associated blood hormone concentrations change. These changes are mostly in the direction of appetite stimulation and persist for at least 12 months. ${ }^{4}$ The adverse effects of excessive nutrient intake extend not only to the obese. Insulin resistance secondary to positive energy balance can affect normal-weight (NW) people leading to cardiovascular disease, type- 2 diabetes, hypertension, and immune disorders. A system for regulating nutrient intake that does not involve dietary restraint is called for.

\section{Historical perspective on the role of hunger in food intake regulation}

Hunger is an internal stimulus that is influenced by food intake. Eating in response to hunger has been regarded as homeostatic and an intermediary step in the process of food regulation. ${ }^{5}$ Workers in psychiatry, psychology, and pediatrics have independently observed that many people eat in response to stimuli that are not influenced by food intake. Stimuli that are not influenced by food intake may override or obscure hunger and include compelling extrinsic factors such as highly palatable and heavily marketed food, ${ }^{6}$ social factors such as eating in the presence of others, ${ }^{7}$ and intrinsic factors such as emotions. ${ }^{8-11}$ Furthermore, people often interpret a wide range of nonspecific body sensations as "hunger."12 These sensations, poorly identified as an undifferentiated unpleasant feeling, could include nausea, pain, or thirst. They can be particularly confusing since they may temporarily disappear after food intake giving the false notion that they represent hunger.

Debate continues as to whether the significance of the hunger sensation must be learned from parents or others, or whether it is instinctive. Thus "eating in the absence of hunger" is thought by some workers to result from imperfect parental instruction. ${ }^{12-14}$ Others regard hunger as instinctive ("intuitive eating") but forestalled by too frequent eating and therefore unrecognized, not reinforced, and eventually forgotten during childhood development. ${ }^{15,16}$ Whatever may be the cause, confusion derived from early childhood about the perception of hunger is prevalent. In the authors' view, this is exacerbated by the widespread practice of imposing scheduled meals in disregard of infants' food-seeking behavior. Habits of food intake, once laid down in infancy, tend to persist and the habit of eating at set times without evaluation of current energy availability becomes "normal" behavior. Thus, even though it may prima facie seem selfevident that adults know whether they are hungry or not, in practice many people assume they are hungry when physiologic hunger is not present, do not perceive physiologic hunger when it is present, or they may confuse it with some other experience such as nausea, anxiety, pain, or thirst. The authors' investigations indicate that hunger corresponds to a different physiological condition to that of appetite (the desire to eat) alone. The desire to eat may occur in the absence of hunger, but hunger alone represents a state of physiological preparedness to digest.

\section{Identifying hunger}

To help people recognize whether they are indeed physiologically hungry, two complementary lines of enquiry were independently followed. Lovell-Smith et al sought to define the subjective hunger experience precisely and hence help people to differentiate between hunger and other experiences. ${ }^{17}$ Building on the early work of Cannon and his contemporaries, hunger was identified as two sets of subjective experiences. The first is the Empty Hollow Sensation (EHS). This corresponds to Cannon and Washburn's hunger pang, is a physical sensation experienced in the epigastrium, and indicates readiness to digest. ${ }^{18}$ This physical epigastric sensation is associated with Phase III contractions of stomach and duodenum. ${ }^{19}$

During the interdigestive period the distal stomach is involved in a recurrent pattern of contractions known as the 
migrating motor complex. ${ }^{20}$ Phase III contractions, the final phase, are complex powerful constrictive and propulsive movements that occur in the distal stomach and duodenum during the intermeal interval. It has been suggested that these contractions have a "housekeeping" effect in clearing the stomach and small intestine of undigested food particles, secretions, debris, and microbes thus readying the subject for food intake. Phase III contractions are likely to be regulated by gut hormones somatostatin and motilin.

The second subjective physical experience often associated with hunger is termed inanition. This includes fatigue, light-headedness, and general weakness. Inanition is taken to indicate the need for nutrition, but not necessarily readiness to digest. As indicated above, both the EHS and inanition are accompanied by, but are distinct from, appetite, the desire to eat. The desire to eat, when it occurs in the absence of EHS or inanition may be prompted by any of the confounding intrinsic or extrinsic stimuli alluded to in the above introduction. The term IH was coined by author $\mathrm{MC}$ and is either EHS or inanition at their first appearance. IH correlates well with BG below about $81.8 \mathrm{mg} / \mathrm{dL}$ $(4.54 \mathrm{mmol} / \mathrm{L}){ }^{21}$ These two approaches to the study of hunger, subjective clarification, and the discovery of an objective marker overlap and are mutually supportive.

\section{The relationship between hunger and BG}

Transient declines in BG pre-meal are well recognized, ${ }^{22-24}$ and $\mathrm{BG}$ decline is associated with hunger. ${ }^{25}$ Glucose has long been considered a prime candidate in the regulation of energy metabolism, being an exclusive energy source for the central nervous system and having limited storage, high turnover rate, and tight regulation. The initiation of hunger and eating behavior is the result of a complex interplay between genetic, psychological, neurological, biochemical, and hormonal factors. Early single factor depletion models such as the glucostatic hypothesis of Mayer ${ }^{26}$ in which it is assumed that all physiology and behaviors seek to maintain a stable glucose have given way to models that recognize this interplay. Nevertheless, BG is a useful metabolic marker in training subjects to recognize hunger. BG is taken to indicate the body's supply of readily available nutrients and their ability to quickly provide energy to body tissues. In a physiologically normal, nondiabetic population, BG therefore indicates the dynamic state of energy balance. High $\mathrm{BG}$ indicates immediate positive energy balance, while low $\mathrm{BG}$ indicates immediate negative energy balance. It should be noted that subjects can be trained to recognize hunger without
BG measurement. This method has been successfully used clinically by one author (DLS) in treating obesity, improving BG control in diabetes mellitus, ${ }^{27}$ and in the management of some autoimmune diseases (see Implications and Future Directions, below); however, further work needs to be done to establish its effectiveness in comparison to concurrent BG measurement. Training without BG measurement is simpler to arrange and does not subject the patient to invasive finger pricks. On the other hand, individuals respond in a multitude of ways to the question: "What do you feel when you are hungry?" Interpretation of these responses requires considerable skill from the trainer/therapist. Thus, although protocols are under development, this method has the disadvantage that training is more difficult to standardize. Concurrent BG measurement provides an objective standard against which subject and trainer/therapist can assess the correspondence of future interoceptive sensations. This is helpful in building confidence in those sensations that subjects are learning to interpret as hunger, it helps "slow learners" to find the relevant sensations more readily, it gives the trainer/therapist an objective "window" into the subject's internal world, it helps the trainer/therapist assess compliance, and helps subjects to correct occasional errors of perception even months or years after training. All formal studies reported here have used the concurrent BG method of training.

\section{Pediatric studies: significant reduction in infant diarrhea} Subjective assessment of hunger by carers

The initial impetus for these studies was to identify feeding patterns that might assist infants in recovering from malnutrition. ${ }^{28}$ Later studies were directed to infants with troublesome diarrhea. MC questioned whether perhaps infants were being overfed or fed at scheduled times, not necessarily when hungry, and were therefore frequently in positive energy balance. In early attempts (pre-1984) to recognize hunger in infants, carers were trained to recognize characteristic signals such as crying (in the first months of life), mood changes including loss of enthusiasm for playing, and gestural or verbal requests for food, or searching for food without any external stimulus. Meals were initiated only when such signals were exhibited (treated infants). Using this method, total energy intake, and days with diarrhea among treated infants decreased significantly, while malnourished infants recovered body weight. ${ }^{29}$ In a further study, compared with 73 control infants, energy intake and days with diarrhea among 70 treated infants decreased significantly. ${ }^{30,31}$ 


\section{Correlation of identified hunger with low BG concentration in infants}

In the latter study, consent was obtained to gather preprandial BG measurements in treated infants to ensure that the "on demand" feeding regime did not put these infants at risk of hypoglycemia. (It was not considered ethical at that stage to measure BG in the control infants as no change had been made to their feeding regimen). It was noted as an incidental finding that the 70 treated infants showed a significant decrease in mean pre-meal BG compared to pretreatment. These preliminary findings argued for a correlation between food demand and preprandial BG and were the stimulus for later adult studies in which BG was measured in both treated and control groups.

\section{Adult studies}

\section{Correlation of $\mathrm{IH}$ and BG}

The method used in all reports is detailed here. Subjects were asked to withhold food intake for a few hours, and make note of the physical sensations that they experienced and which they associated with the need for food intake. The EHS was the most frequent and recognizable sensation, followed by inanition. After determining their symptoms, subjects were trained to measure their own BG by portable glucometer and thus learned to associate these sensations with BG concentration. Subjects were encouraged to eat only when the sensations of IH were present. The principal investigator phoned each subject at the end of the first day of training to ascertain any changes made by the subject in eating pattern. A mean delay of 2 hours (range $0-48$ hours) in meal intake was noted on the first day of training. At subsequent mealtimes, adults evaluated their physical sensations according to the BG-correlated experience to help them assess if their present sensation was indeed hunger or whether their desire to eat was conditioned by time of day or other extrinsic factor. Subjects were encouraged to become familiar with the hunger sensation and to take a meal only at its first appearance (see Appendix).

The effectiveness of this method in altering eating habits needs to be spelled out to be fully appreciated. In effect, subjects stopped eating "automatically." This means that they stopped eating according to extrinsic cues and began eating according to their intrinsic interoceptive awareness of hunger. The method encourages subjects to make a judgment on the amount to be eaten such that hunger will appear at the next mealtime. This is a radically different approach to other systems of dietary instruction in which emphasis is laid upon feelings of satiety. In the authors' experience, subjects find instructions such as "stop before you feel full" and the like restrictive and disheartening. Such instructions tend to be ignored leading to excessive food intake. By contrast the instruction "start when you feel empty" invites compliance, since eating when hungry is inherently pleasurable. For most subjects at the beginning of training, a meal intake of $150 \mathrm{kcal}$ for infants and $300 \mathrm{kcal}$ for adults ensured hunger at the subsequent mealtime, with adjustments spontaneously made in either increasing or decreasing energy-dense food to reach IH at the desired time. In spite of a generally lower caloric intake subjects reported normal activity and did not report lowered energy during the intermeal period.

Subjects received information on food energy content, recommended vegetable intake, and physical activity per day. The recommended fruit and vegetable amount for adults was $1 \mathrm{~kg} /$ day. The investigators recommended avoiding high ambient temperatures and wearing excessive clothing as these tend to slow metabolic rate and delay the appearance of hunger sensations. They also encouraged normal outdoor and gym activities. Avoiding snacks was suggested, though earlier than optimal IH was satisfied with fruit and adequate energy-dense food if needed. Social obligations such as parties and school catering were included in planning the intake amount and timing of the previous and subsequent meals.

Interaction between expert and subject was necessary for most subjects to interrupt automatic feeding and to stop any reliance on fullness sensations toward meal end. The process generally became "second nature" after a few days. The subject realizes that $\mathrm{IH}$ will arise at the desired times when intake is accurately judged. The intervention may be summarized as an effortless cessation of automatic feeding and its replacement by an informed judgment on the amount to be eaten.

\section{Is self-estimation of BG concentration possible?}

If hunger and low BG are correlated, then using the above method should make it possible for a subject to predict his or her BG by reference to hunger sensations alone. This was demonstrated by Ciampolini and Bianchi. ${ }^{32}$

In this randomized controlled trial, 158 adults were randomly assigned to experimental (trained, $n=80$ ) and control (untrained $n=78$ ) groups. Over 7 weeks the experimental group were trained to measure pre-meal blood glucose by glucometer at the earliest feelings of hunger or discomfort (hence the term "IH"). A 7-week period was chosen to ensure a high degree of facility in the IH Meal Pattern; however, the majority of subjects learned the skill within 2 weeks. Subjects were asked to state whether they were or were not feeling hunger sensations and then estimate on that basis their pre-meal glucose concentration. Control subjects 
were asked to estimate their glucose levels by referring to a range of values between the extremes of $60 \mathrm{mg} / \mathrm{dL}$ ("intense hunger") and $100 \mathrm{mg} / \mathrm{dL}$ ("after a satiating meal"). Blood samples were taken from each group.

Figure 1 shows that even though members of the control group considered themselves hungry, they were unable to reliably estimate their blood glucose concentration whereas members of the trained group, who considered themselves hungry, were able to do this with remarkable accuracy. The correlation lines for the "hungry" groups in Figure 1 show that there was significant correlation between estimated and measured BG in the trained group $(\mathrm{r}=0.92 ; P=0.0001)$ but not in the control group $(\mathrm{r}=0.29 ; P=0.06)$. The subjective experience of hunger thus appears for most people to be reliably associated with the objective marker BG, but only when people are trained to recognize hunger.

Not all subjects reported the EHS in the final session. All trained and control subjects who did not experience the EHS estimated their BG to be significantly lower than measured, with the control subjects performing more poorly than trained (the estimation errors being $4.8 \% \pm 3.2 \%$ and $16.1 \% \pm 11.3 \%$ in the trained and control groups, respectively). The linear correlation between estimated and measured BG was highly significant in the trained group that did not experience the

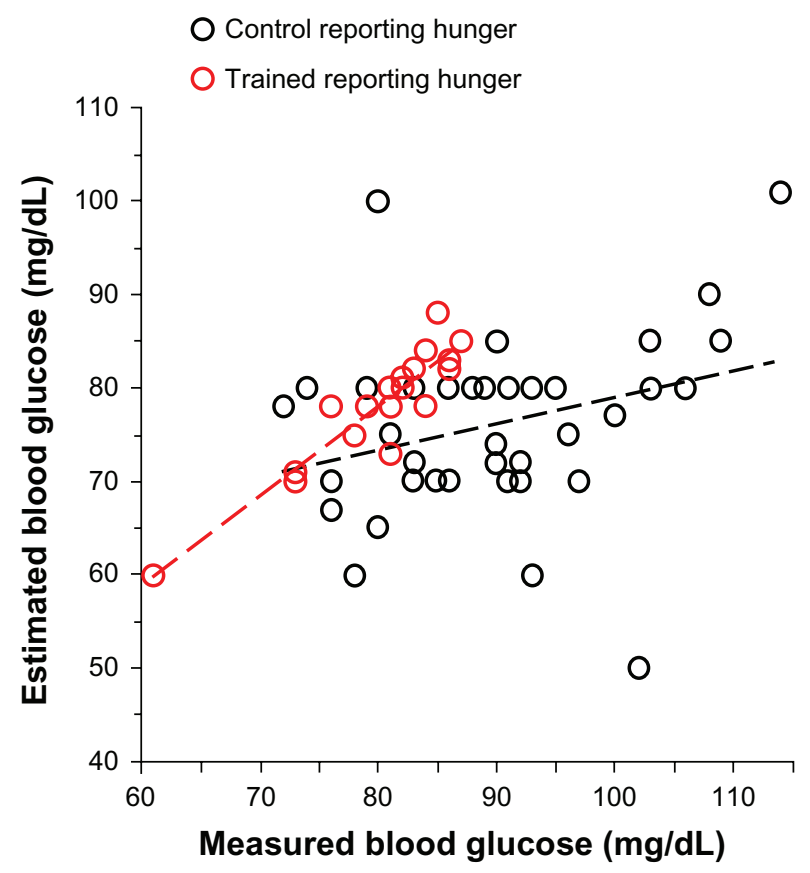

Figure I Estimated versus measured blood glucose of trained subjects (hollow red circles; $n=18$ ) and control (untrained) subjects (hollow black circles; $n=42$ ) reporting to be hungry at the final laboratory investigative session.

Notes: Linear correlation was significant for the trained data (red dashed line; $r=0.92 ; P=0.000 \mathrm{I}$ ) but not for the control data (black dashed line; $r=0.29, P=0.06$ ). Image courtesy of Ciampolini M, Bianchi R. Training to estimate blood glucose and to form associations with initial hunger. Nutr Metab (Lond). 2006;3:42. ${ }^{32}$
EHS $(\mathrm{r}=0.68 ; P=0.0001)$ and not significant in controls who did not experience the EHS $(\mathrm{r}=-0.12 ; P=0.32)$. This difference can be accounted for by trained subjects' reports that they were able to recognize subtle cues other than the EHS, which they found useful in estimating glycemic levels. These subtle cues included feelings of physical weakness, difficulty concentrating, impatience, irritability, drowsiness, and loss of enthusiasm and are the symptoms that are termed inanition. In other words, recognizing inanition allowed trained subjects to achieve similar accuracy in estimating BG as those trained subjects who recognized the EHS (Figure 2).

Is inanition hunger then? It has been included it in the definition of IH because the authors consider both the EHS and inanition are valid signals to start a meal. Do they carry equal significance physiologically? Probably not, since only the EHS is accompanied by Phase III gastrointestinal tract contractions. Although further work is needed to elucidate the full significance of these signals, it seems likely that the EHS indicates a readiness to digest that is not necessarily

Trained reporting EHS

- Trained not reporting EHS

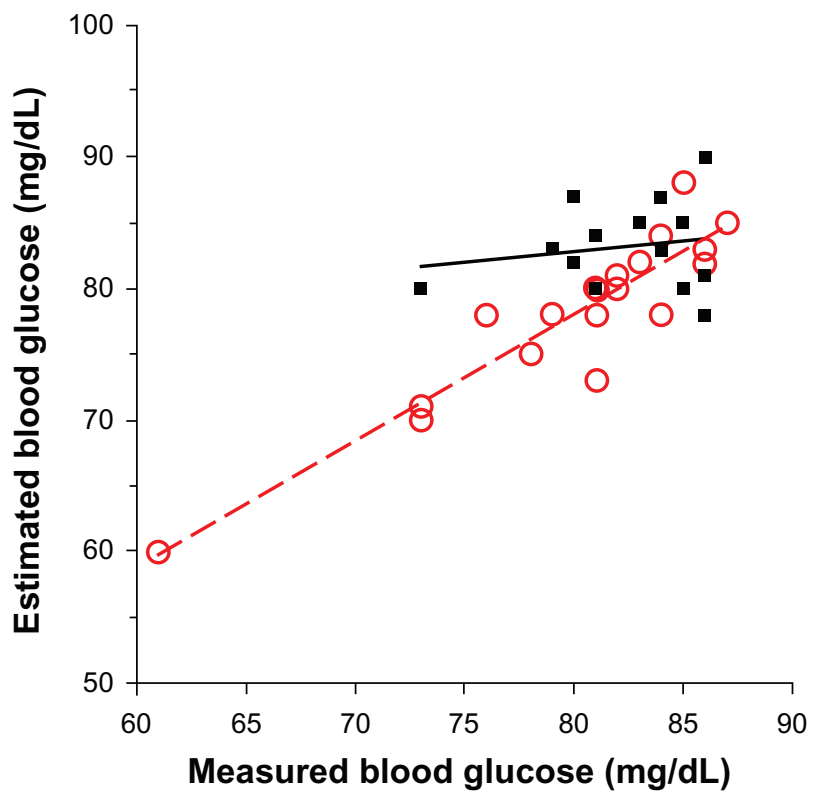

Figure 2 Estimated versus measured blood glucose of trained subjects with measured blood glucose $<87 \mathrm{mg} / \mathrm{dL}$ at the final session.

Notes: Below this value, 18 subjects reported the EHS (hollow red circles) and 14 subjects reported inanition only but not EHS (filled black squares). These 14 subjects showed an average estimation error of $4.5 \% \pm 3.1 \%$ of the measured blood glucose, which did not significantly differ from the estimation error of the 18 trained subjects who reported the EHS $(3.2 \% \pm 2.4 \% ; P=0.20)$. Linear regression was significant for the hungry subjects reporting EHS (dashed red line; $r=0.92$; $P=0.000 \mathrm{I}$ ) but not for those not reporting EHS (solid black line; $r=0.18 ; P=0.54$ ). Image courtesy of Ciampolini M, Bianchi R. Training to estimate blood glucose and to form associations with initial hunger. Nutr Metab (Lond). 2006;3:42.32

Abbreviation: EHS, Empty Hollow Sensation. 
indicated by inanition. In the clinical setting, DLS has found it advantageous to recommend light meals in response to inanition.

To the authors' knowledge, this study on the relationship between BG and hunger is the first in over 60 years to refer research subjects to their specific subjective symptoms. Intervening studies, such as that by Bernstein and Grossman, that appear to show a lack of correlation between low premeal blood glucose and hunger used hunger questionnaires and hunger rating scales and were therefore subject to all the limitations of subjects' untrained interoceptive perception. ${ }^{33}$ In the authors' experience in untrained subjects, adults' hunger is related to a particular metabolic condition in only a small number of people. Such studies led to a general perception that while the brain might be able to detect gross changes in BG (eg, many diabetic patients can perceive extreme hypoglycemia), it is unable to detect more subtle changes. ${ }^{34}$ By contrast, this study demonstrates that with training, people can estimate their pre-meal blood glucose with accuracy and hence estimate their current energy availability and energy requirement.

\section{Establishment of a hunger-based meal pattern}

Once the subjective sensation of hunger has been recognized, the measurement of BG functions as an objective marker against which the subject can check ongoing pre-meal subjective sensations. It should be noted that subjects are not asked to wait until BG is low or at its nadir before checking their hunger sensations. On the contrary, the subject first notes the subjective experience then checks it against BG. BG acts to clarify, verify, and validate what might otherwise remain as undifferentiated interoceptive sensations.

After a few days of trial and error and sometimes irregular mealtimes, $\mathrm{MC}$ found subjects were able to arrange their meal size and composition to ensure that IH appeared just prior to the following meal-time with a mean error of half an hour in $80 \%$ of adults and $90 \%$ of children. This is the pattern of food intake that has been called the IH Meal Pattern.

The next step was to investigate the metabolic consequences of sustained application of this meal pattern. The initial aim was to see whether by creating immediate blood energy balance (avoiding food intake in the presence of high BG and taking food only in the presence of low BG) it would be possible to balance total body energy intake to energy requirement long term, ie, to achieve total body energy balance as shown by neither increase or decrease of body weight in NW subjects and loss of weight in OW subjects, over a period of months. ${ }^{35}$
It was also desired to see whether creating immediate blood energy balance would improve insulin sensitivity. ${ }^{36}$

\section{Recognizing hunger before each meal: metabolic consequences}

A total of 181 subjects aged 18-60 years were recruited between the years 1995 and 2000. All subjects entered a randomized controlled study in which the primary outcome was weight. ${ }^{35}$ One-hundred and forty-nine subjects completed the full study. One-hundred and forty-three subjects were included in a second study whose primary outcome was insulin sensitivity. ${ }^{36}$ All subjects were trained in Hunger Recognition over a 7-week period then followed for a further 3 months.

\section{Weight}

Hunger Recognition was associated with significant decreases in body weight and body mass index (BMI) in OW subjects compared to controls after 7 weeks of training and after 3 further months of application. BMI decreased from $28.7 \pm 3.5$ to $26.5 \pm 3.5$ in the trained group. The decrease was significant in comparison to controls $(P=0.004)$ and in comparison with baseline values of the same group $(P=0.0001)$. Multivariate analysis of variance showed a significant association between training and both BMI and weight. Pre-meal BG emerged as the most significant predictor of variations in BMI and body weight. NW subjects maintained weight overall; however, those trained NW subjects whose BG was high at recruitment also lost weight compared to controls.

\section{Insulin sensitivity}

Hunger Recognition was associated with significant decreases in insulin and BG peaks, insulin at 60 minutes and 90 minutes during the glucose tolerance test, glycosylated hemoglobin, and mean pre-meal BG as well as energy intake and BMI when compared to controls. Insulin sensitivity index increased from $7.1 \pm 4.1$ to $9.4 \pm 5.2$. The increase was significant in comparison to controls $(P<0.01)$ and in comparison with baseline values of the same group $(P<0.001)$.

\section{Post hoc analysis}

In the latter study it was noticed that at recruitment mean pre-meal BG ranged from $64.5 \mathrm{mg} / \mathrm{dL}$ to $109.9 \mathrm{mg} / \mathrm{dL}$. This wide distribution suggested that the overall improvement in insulin sensitivity at study end could be mostly accounted for by improvements in those subjects whose pre-meal BG was high at recruitment. Furthermore, it was noted 
that for the week in which each subject kept a diary of pre-meal BG, the mean confidence interval (95\%) around that subject's mean pre-meal BG was only $\pm 3.84 \mathrm{mg} / \mathrm{dL}$. Thus, although subjects differed widely from each other in BG concentration pre-meal, their own individual pre-meal BG concentration varied little. This suggests that many people eat at an arbitrary BG, not necessarily according to homeostasis and more likely associated with habit. For many subjects, energy intake was habitually high and was likely leading to positive energy balance. Other subjects were already eating at low BG concentration and thus probably already eating more or less according to homeostasis. To test this, the effect of Hunger Recognition on each of two subgroups - those who at recruitment were found to eat habitually at a high blood glucose concentration (high BG) and those who at recruitment habitually ate at a low blood glucose concentration (low BG) - was analyzed. The demarcation point that divided the two groups was a mean pre-meal BG concentration of $81.8 \mathrm{mg} / \mathrm{dL}$ ( $4.54 \mathrm{mmol} / \mathrm{L}$ ). This was the $B G$ value that statistically most significantly divided the two groups.

\section{Stability of mean pre-meal BG among control low BG and control high BG subjects}

Pre-meal BG among control subjects did not significantly change during the study. Thirty-one control subjects, comprising members of both high BG and low BG groups, maintained a stable mean pre-meal BG after 5 months (from $85.2 \pm 8.1 \mathrm{mg} / \mathrm{dL}$ to $85.3 \pm 7.6 \mathrm{mg} / \mathrm{dL}$ ). The absolute pre/ post change (increase or decrease) was $6.0 \pm 4.6 \mathrm{mg} / \mathrm{dL}$ with a confidence interval (95\%) from $3.1 \mathrm{mg} / \mathrm{dL}$ to $8.9 \mathrm{mg} / \mathrm{dL}$. This confirmed the suspicion that left to their own devices, untrained subjects continue to eat habitually at a given pre-meal BG concentration which may or may not be in accordance with homeostasis.

\section{Variation in mean pre-meal BG among trained low BG and high BG subjects}

High BG trained subjects significantly decreased their mean pre-meal BG $(\mathrm{n}=55$; pre: $91.6 \pm 7.7 \mathrm{mg} / \mathrm{dL}$; post: $81.0 \pm 7.7 \mathrm{mg} / \mathrm{dL} ; P<0.0001$; Figure 3). Among low BG trained subjects $(\mathrm{n}=34)$ pre-meal $\mathrm{BG}$ remained relatively constant (pre: $76.6 \pm 3.7 \mathrm{mg} / \mathrm{dL}$; post: $77.2 \pm 4.2 \mathrm{mg} /$ $\mathrm{dL} ; P=0.499)$. Trained high BG but not low BG subjects showed a cumulative energy balance that was negative over the 5 months of the study as indicated by measurement of skin-fold thickness. Furthermore, trained high BG but not

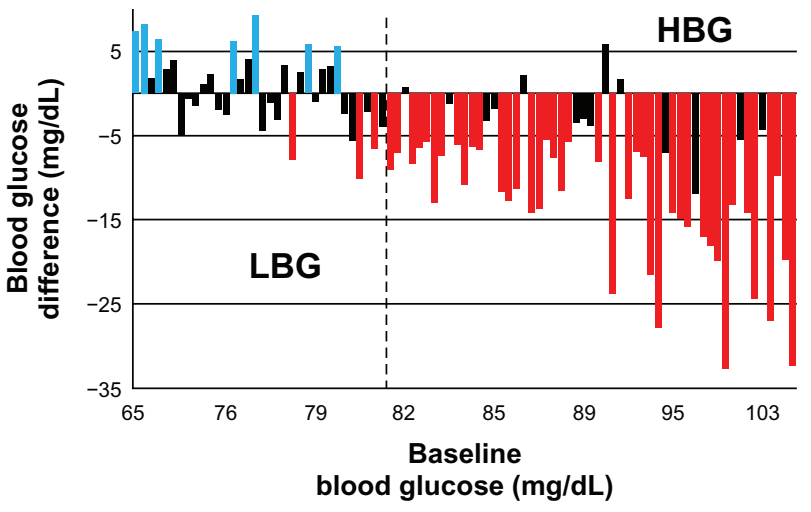

Figure 3 Difference of mean preprandial BG after training versus BG at recruitment for each trained subject.

Notes: Column height shows 5 months after pretraining mean BG difference in each trained subject. Significant increases are indicated by blue bars, significant decreases by red bars, and not significant changes by black bars. Mean BG is reported in sequentially increasing order at recruitment, not in linear correlation with segment length on the $\mathrm{X}$-axis scale. The range of mean blood glucose values at recruitment is indicated by the minimum and maximum values for the first and last subjects (large arrows). The vertical dashed line indicates the most significant division between subjects who showed no mean BG decrease after training (low BG group, $n=34$ ) and those who showed significant decrease of mean BG (high BG group, $\mathrm{n}=55$; Chi-squared analysis: $P=0.0000 \mathrm{I}$ ). This threshold blood glucose at recruitment (demarcation point) is $81.8 \mathrm{mg} / \mathrm{dL}(4.5 \mathrm{mmol} / \mathrm{L})$ at recruitment in the randomized controlled studies. Subjects above this threshold accounted for most of the improvements in weight and insulin resistance. ${ }^{35,36}$

Copyright (c) 201I, Dove Medical Press Ltd. Reproduced with permission from Ciampolini M, Sifone M. Differences in maintenance of mean blood glucose (BG) and their association with response to "recognizing hunger." Int J Gen Med. 20I I; $4: 403-412 .^{21}$

Abbreviations: BG, blood glucose; HBG, high blood glucose; LBG, low blood glucose.

low BG subjects showed decreased insulin area under the curve, an index of whole body insulin resistance, decreased glycosylated hemoglobin, and increased insulinogenic index, ${ }^{37}$ suggesting that low pre-meal BG is physiologically appropriate for energy needs. Only those subjects whose energy intake (and BG) was high at recruitment adjusted their food intake after training. Those for whom energy intake was already low made little or no adjustment. These data are consistent with homeostasis and support the notion that the observed effects could be accounted for mainly by the effect of the intervention on the high BG trained subjects.

\section{Variation in weight among trained low BG and high BG subjects}

In post hoc analysis of the study on weight, subjects were divided into four groups according to weight (OW; NW) based on BMI and pre-meal BG at recruitment. The four groups were thus: OW high BG, OW low BG, NW high $\mathrm{BG}$, and NW low BG. As might be expected, after training OW high BG and OW low BG subjects lost weight and NW low BG subjects retained weight, further confirming the homeostatic nature of Hunger Recognition. However, NW high BG subjects also lost weight. ${ }^{35}$ The distinction 
between NW and OW based on BMI is, after all, arbitrary. It is concluded that some subjects with pre-meal high BG but so-called "NW" may, in fulfillment of homeostatic requirements, need to lose weight. For this situation, the term "hidden fattening" is suggested. This term is chosen since it conveys the notion that many of the risks of obesity are, in reality, the risks of insulin resistance. Thus, NW subjects with insulin resistance are no less subject to the risks of obesity than the obese. It follows that mean pre-meal BG and insulin sensitivity may be better predictors of risk than weight or BMI calculation.

\section{Limitations}

\section{Limitations in the studies}

The main limitation in the latter two studies was the high dropout rate among subjects. However, as detailed in the reports, sensitivity analysis suggests that the dropout population did not significantly differ from the population who completed the study. It is also acknowledged that owing to the limitations imposed by post hoc analysis, the conclusions with respect to subgroups are indicative rather than conclusive.

\section{Limitations to BG-hunger correlation}

The statistical analysis suggests that a demarcation point between homeostatic eating and nonhomeostatic eating occurs at a BG concentration of around $81.8 \mathrm{mg} / \mathrm{dL}$ (4.54 mmol/L). It is tempting to conclude that hunger always occurs below this BG and not above, and that homeostatic eating can be ensured by eating only when one's BG is below $81.8 \mathrm{mg} / \mathrm{dL}$. However, there does appear to be possible variation in the $\mathrm{BG}$ at which physical hunger appears and hence variation in the pre-meal BG that might be considered a correct eating prompt for a given individual. After 7 weeks of training, 77 of 89 trained subjects recognized hunger at a mean pre-meal BG of $<81.8 \mathrm{mg} / \mathrm{dL}$ and 62 maintained low pre-meal BG at the end of the investigation. Of the 27 remaining who showed high $B G$ at the end of the investigation, 15 had shown low BG at the end of their 7-week training period. Their regression to high BG could be attributed to fading compliance with their instructions in the final weeks of investigation. The twelve subjects with high pre-meal BG reported hunger did not achieve low BG after training. Of these, six were involved in heavy manual work for 8-10 hours per day in cold ambient conditions. These subjects' reports, their insulin, BG area under the curve, and insulin sensitivity index at the final examination suggested they complied with the IH Meal Pattern. They reported hunger, yet their mean pre-meal BG was $86.4 \pm 4.0 \mathrm{mg} / \mathrm{dL}$, higher than the statistically derived demarcation point of $81.8 \mathrm{mg} / \mathrm{dL}$. The demarcation between compliance and noncompliance with Recognizing Hunger is statistically strong at $81.8 \mathrm{mg} / \mathrm{dL}$, but subjects with high energy expenditure might comply with Recognizing Hunger and yet show higher pre-meal BG concentration than $81.8 \mathrm{mg} / \mathrm{dL}$. This conclusion is supported by the significantly higher insulin sensitivity shown by the six manual workers when compared to the other 21 subjects (Table 1). At study end they showed higher insulin sensitivity than the other 21 subjects in the face of a pre-meal BG at study end that was statistically no different from that of the other 21 subjects $(86.4 \pm 4.0 \mathrm{mg} / \mathrm{dL}$ for the six high mean BG subjects and $87.1 \pm 5.3 \mathrm{mg} / \mathrm{dL}$ for the 21 high BG subjects; Table 1). These observations suggest that a similar association might be found with other physiological conditions such as fever and snacking (ie, intake of small amounts of food within an hour of BG measurement).

\section{Implications and future directions of the work Weight loss \\ Avoids restraint}

Conventional dieting for weight loss generally involves restraint in food intake whose long-term efficacy is poor. In the study on weight, control subjects were encouraged to lose weight in the conventional way. Control OW high BG subjects decreased energy intake and had indeed significantly

Table I Effects of heavy outdoor work ${ }^{\dagger}$ in 27 trained subjects who remained with high blood glucose at investigation end

\begin{tabular}{lcc}
\hline & 6 HBG $^{\dagger}$ & 2 I HBG \\
\hline Mean BG $(\mathrm{mg} / \mathrm{dL})$ & $86.4 \pm 4.0$ & $87.1 \pm 5.3$ \\
Final insulin AUC $\left(\mathrm{mU} \mathrm{L}^{-1} 3 \mathrm{~h}^{-1}\right)$ & $124 \pm 26$ & $207 \pm 99 * *$ \\
Final BG AUC $\left(\mathrm{mg} \mathrm{dL}^{-1} 3 \mathrm{~h}^{-1}\right)$ & $536 \pm 56$ & $601 \pm 82^{*}$ \\
Insulin sensitivity index & $1 \mathrm{I} .4 \pm 2.9$ & $6.68 \pm 4.0^{* * *}$ \\
Beta-cell function index & $1.29 \pm 0.66$ & $1.43 \pm 1.22$ \\
\hline
\end{tabular}

Notes: Values are expressed as mean \pm standard deviation. $* P<0.05 ; * * P<0.01$; $* * * P<0.001$ (Student's $t$-test); † six HBG subjects reported full days of heavy manual labor in an outdoor environment during a cold winter season. Their reports and measurements suggested that they had complied with the Initial Hunger Meal Pattern. There were no significant differences in the five above parameters at study end from recruitment (mean BG at recruitment $=86.9 \pm 5.3 \mathrm{mg} / \mathrm{dL}$ ). At study end they showed higher insulin sensitivity than the other $2 \mathrm{I}$ subjects in the face of a pre-meal BG at study end that was statistically no different from that of the other 21 subjects $\left(87.1 \pm 5.3 \mathrm{mg} / \mathrm{dL}\right.$ for the 21 subjects); ${ }^{\text {t the }} 21 \mathrm{HBG}$ subjects included I 5 that were low BG after 7 weeks training (at clinical assessment) and six who had higher mean BG than $100 \mathrm{mg} / \mathrm{dL}$ at recruitment.

Copyright (c) 201I, Dove Medical Press Ltd. Reproduced with permission from Ciampolini M, Sifone M. Differences in maintenance of mean blood glucose (BG) and their association with response to "recognizing hunger." Int J Gen Med. 20I I; $4: 403-412 .^{21}$

Abbreviations: AUC, area under the curve; BG, blood glucose; HBG, high blood glucose. 
lost weight at study end from $77.1 \pm 16.2 \mathrm{~kg}$ to $72.8 \pm 15.3$ ( 7 weeks) to $73.7 \pm 15.9 \mathrm{~kg}$ (5 months). However, all significant weight loss took place in the first 7 weeks in these subjects. Their energy intake during the final weeks of the study rose significantly (from $1082 \pm 291 \mathrm{kcal} /$ day at 7 weeks to $1343 \pm 467 ; P=0.02$ at 5 months), suggesting dietary disinhibition.

\section{Not onerous}

It is well recognized that food tastes delicious when one is hungry. ${ }^{38}$ This provides positive reinforcement for compliance with the IH Meal Pattern. Subjects report that it is not onerous. Compliance is good. OW subjects reported their hunger was of no greater intensity nor was it more prolonged than NW subjects. These findings suggest that Hunger Recognition is effective in promoting weight loss and provides an appealing alternative to dietary restraint. In contrast to most dietary systems, this method eliminates the need to identify sensations of fullness at meal end, a process that is disheartening and also deceptive, especially for OW subjects who have high insulin release during meals in the first weeks of intake limitation and in whom feelings of satiety are therefore delayed.

\section{Meal by meal adjustment of food intake}

In attempting to lose weight, rather than seeking an arbitrary and often daunting goal weight, subjects following the $\mathrm{IH}$ Meal Pattern receive immediate meal-by-meal feedback on food intake from their physiological hunger signals. Food intake is adjusted to accommodate hourly changes in energy need. Subjects thus eat only when food is required for energy. Subjects are protected from overconsumption but are also protected from taking insufficient energy for day-to-day body functioning as may occur with restraint diets of $<900 \mathrm{kcal}$ per day. The guilty temptation to eat more while "on a diet" is removed, which helps provide a stress-free approach to eating. Subjects are also able to find their own physiological NW rather than pursue an arbitrary statistical norm.

\section{Solution to hunger rating scale woes}

The difficulty that untrained subjects have in recognizing hunger calls into serious question these subjects' ability to provide accurate or useful data in completing traditional hunger rating scales. This casts doubt on the findings of the many studies in which such rating scales have been used. It must be questioned whether hunger rating scales have been either sensitive (hunger might be present but missed as in anorexia) or specific (hunger is frequently not present but reported). ${ }^{39-41}$ After Hunger Recognition training, the response to the question "Are you hungry?" is more likely to be a reflection of the underlying physiological state than before training, and thus of greater use to the investigator. It should be noted that in training people to recognize the subjective symptoms EHS and inanition, supported by objective BG measurement, no rating scales or visual analog scales are used. Subjects do not rate hunger, but rather recognize it. A person either experiences the EHS or does not. This "either/or" response is, in the authors' experience, more useful than the degree to which it is experienced. EHS arises as a threshold phenomenon, although inanition arises gradually.

\section{New approach to diabetes prevention and treatment}

Type 2 diabetes mellitus is a devastating outcome of maintaining positive energy balance. Hunger Recognition suggests an innovative approach to diabetes prevention and control. In a longitudinal investigation of 13,163 subjects, a fasting plasma glucose of $\geq 87 \mathrm{mg} / \mathrm{dL}$ ( $3.9 \mathrm{mmol} / \mathrm{L})$ was found to be associated with an increased risk of noninsulin-dependent diabetes mellitus in men compared to those whose fasting plasma glucose was $<81 \mathrm{mg} / \mathrm{dL}(4.5 \mathrm{mmol} / \mathrm{L}) .{ }^{42}$ It is interesting that this latter figure corresponds closely to the demarcation point which separated the current study's low BG and high $\mathrm{BG}$ groups. By recognizing hunger pre-meal and eating only when hunger is present, most of the subjects were eating at a $\mathrm{BG}$ of $<81 \mathrm{mg} / \mathrm{dL}$. The finding that the high BG control subgroup showed a decreased insulinogenic index of beta-cell function whereas the high BG trained subgroup showed an increase in this index supports the use of Hunger Recognition in diabetes prevention and treatment. The difference in insulinogenic index between the two groups was significant, implying higher insulin production and preservation of beta-cell function in the trained group. It should be noted that the IH Meal Pattern is diametrically opposite to the prevailing treatment of diabetes which calls for unvarying caloric intake at prescribed times during the day. Diabetes control using this rigid method is notoriously difficult. It is suggested that the meal-by-meal feedback furnished by the IH Meal Pattern is a more physiologic approach to this widespread and difficult disorder. 


\section{Implications of improved insulin sensitivity Resolution of the proinflammatory state}

It is a clinical commonplace that healing is poor in uncontrolled diabetes. However, prediabetic glucose metabolism derangement is also associated with immune disruption and low-degree inflammatory activity. This has been variously termed the proinflammatory state or subclinical inflammation, ${ }^{43-46}$ and is characterized by the development of bacterial biofilms (including Helicobacter pylori) inside the alimentary canal. Subclinical inflammation is implicated in the gradual development of cardiovascular disease, cancer, autoimmune diseases, and other disorders. ${ }^{47} \mathrm{In}$ a randomized controlled study, Ciampolini et al studied the direct effect of Hunger Recognition on H. pylori gastrointestinal disease. ${ }^{48}$ They trained a group of children and adults $(\mathrm{n}=24)$ aged 60 months to 25 years known to have $H$. pylori serum antibodies to recognize hunger (recovery study). Twentythree subjects of similar age and serum antibodies formed the control group. The outcome was assessed after about 1 year. They also trained another group of healthy children $(n=43)$ aged 6-60 months in comparison with 43 healthy control subjects of the same age (prevention study). Both groups were assessed at 4,8 , and 12 years postintervention.

The diagnosis of $H$. pylori gastrointestinal disease is supported by the presence of serum antibodies whose titer decreases after successful treatment. In the recovery study, 15 of 24 trained subjects (62.5\%) had lost seropositivity for H. pylori compared to only three of $23(13 \%)$ control subjects $(P=0.002)$. In the prevention study, 13 of 43 control subjects showed seropositivity at study end compared to just two positive (plus one transient) conversions to seropositivity among the intervention group. This difference was highly significant and corresponded to one infection every 176 years under intervention compared to one infection every 25.6 years in control subjects.

Gastric emptying and upper gastrointestinal secretions are known to increase with increasing insulin sensitivity and are inhibited by high glycemic concentrations. ${ }^{49-51}$ Although $H$. pylori thrive at $\mathrm{pH}$ 6-7 (ie, lower than most bacteria), it cannot survive in the extreme acidity commonly found, for example, in the fundus of the stomach. In these conditions, its acid-buffering strategies (such as the production of ammonia by urease) are overcome. H. pylori therefore seek a niche in which $\mathrm{pH}$ and other factors are optimal for its survival. ${ }^{52}$ Reference has already been made to the "housekeeping" effect of Phase III contractions associated with the EHS. Approximately $20 \%$ of $H$. pylori in the stomach adheres to the surfaces of mucus epithelial cells partly to inflict cellular damage and inflammation but also to avoid mechanical clearance. ${ }^{53}$ Gastric and duodenal "sweeping" combined with lowered gastric $\mathrm{pH}$ may account for the inhibition of $H$. pylori proliferation found by Ciampolini et al.

H. pylori is just one of a number of intestinal bacteria that may proliferate when high glycemic concentrations inhibit gastric motility. Ciampolini et al have shown that in children with irritable bowel syndrome and celiac disease, the bacteria count (mainly streptococci and staphylococci) per gram of mucosa for all subjects was 24 times higher after a 20-hour fast than after a 26-hour fast and for celiac sufferers, 39 times higher. In these subjects, bacteria persisted longer than the intermeal interval. ${ }^{54}$ Other immunogenic bacteria are implicated in irritable bowel syndrome, ${ }^{55}$ colorectal cancer, and obesity. ${ }^{56}$ By helping to avoid glycemic peaks, clearing the bowel of unwanted bacteria, and preventing the proinflammatory state, Hunger Recognition may help prevent a wide range of disorders.

These disorders may extend to the autoimmune diseases. Fasting has long been recognized as beneficial in rheumatoid arthritis, ${ }^{57}$ but dismissed as an "impractical management strategy" 58,59 since it is not possible to fast indefinitely. The IH Meal Pattern is, in effect, a controlled fasting in which the subject fasts just long enough to allow hunger to appear. This yields the benefits of fasting without the disadvantages. The inflammatory marker C-reactive protein is one indication of the proinflammatory state and shows a strong positive correlation with insulin resistance. One of the authors (DLS) has noted dramatic and sustained clinical improvement, supported by reversion of C-reactive protein to normal concentrations, among patients suffering from rheumatoid arthritis ${ }^{60}$ and Crohn's disease after training in Hunger Recognition as well as sustained improvement in a case of Graves' disease ${ }^{61}$ Further work is indicated to further investigate the role of recognizing hunger in treating these autoimmune disorders.

\section{May help with psychological stress}

Hormones that allow the body to meet stress such as cortisol, cortisol releasing factor, and serotonin together raise blood glucose concentration, activate mast cells, monocytes, and macrophages, increase intestinal permeability, and contribute to subclinical inflammation - essentially the same effects as eating in the absence of IH. Recognizing hunger may therefore help to reverse the deleterious physiological effects of psychological stress by loss of one or two meals. 


\section{Training should begin early}

Training in Hunger Recognition should begin in infancy and continue through childhood. Young children are commonly enjoined to "eat everything on their plate" at closely scheduled mealtimes with no regard to whether the child is actually feeling hunger. Based on the current findings, this instruction, often given simply for reasons of social convenience, has disastrous implications for the health of the child. Habits of eating learned early tend to persist. It becomes "normal" for the child to be unable to recognize hunger and hence unable to evaluate current energy availability. Food choices tend to be conditioned by external stimuli or emotional internal stimuli and are made "without full conscious awareness." ${ }^{2}$ To manage the weight gain that ensues, the child may then be subjected to the stress of restraint-based dieting. Insulin resistance syndromes have reached pandemic proportions. ${ }^{63}$ They are at the root of the most serious illnesses in Western culture. Early training in Hunger Recognition has the potential to sidestep habitual overconsumption, the root cause of most Western illnesses.

\section{Conclusion}

The last 60 years have seen huge effort towards teasing out the physical, neurological, and biochemical pathways that regulate food intake and energy balance. The motivation generally is to discover drugs that will help foster energy balance. While unraveling physiological complexities has its own fascination, the solution to achieving energy balance may be as simple as asking people to wait before they eat until they experience objectively validated hunger.

\section{Resume}

\section{What was already known}

The role of hunger in regulating food intake for energy balance had been arrived at by a number of disciplines working independently.

\section{What had been overlooked}

Many people are confused about the perception of hunger. People cannot be relied upon to recognize hunger without training. There was a need for greater clarity about the subjective experience of hunger and an objective "hunger marker."

\section{What could not be known}

"What it is like to be hungry" could not be known so long as subjective experience was largely excluded from the scientific domain. Hunger self-report scales were an attempt to "objectify" hunger that has enjoyed only limited success. Returning to hunger as a subjective experience, the authors suggest that subjective hunger can be well defined and recognized using blood glucose concentration as an objective marker of a recurrent physiological condition.

\section{What the authors' studies have added}

Hunger has been differentiated from other misleading and confounding experiences by clarity of definition and by association with an objective marker. An association between the experience of Initial Hunger and low blood glucose has been demonstrated. It has also been demonstrated that Hunger Recognition yields improvements in insulin sensitivity in both normal-weight and overweight subjects and loss of weight in overweight subjects.

\section{Implications}

Hunger Recognition could help people regulate their food intake and thereby achieve energy balance. Hunger-promoted energy balance could help alleviate obesity, diabetes, heart disease, autoimmune disorders, and stress - the great scourges of Western industrialized societies.

\section{Abbreviations}

BMI: body mass index (body weight in $\mathrm{kg} / \mathrm{height}$ in square meters); BG: blood glucose concentration; EHS: empty hollow sensation. The epigastric hunger "pang"; High BG: high mean pre-meal BG (> $81.8 \mathrm{mg} / \mathrm{dL})$; IH: initial hunger. Low BG-associated hunger. The EHS and/or inanition at their first appearance; IH Meal Pattern: a meal pattern in which IH and low BG is present pre-meal for most meals; Low BG: low mean pre-meal BG $(<81.8 \mathrm{mg} / \mathrm{dL})$; NW: normal weight; OW: overweight.

\section{Disclosure}

The authors report no conflicts of interest in this work.

\section{References}

1. Hossain P, Kawar B, El Nahas M. Obesity and diabetes in the developing world - a growing challenge. $N$ Engl J Med. 2007;356(3): 213-215.

2. Mozaffarian D, Hao T, Rimm EB, Willett WC, Hu FB. Changes in diet and lifestyle and long-term weight gain in women and men. $N$ Engl $J$ Med. 2011;364(25):2392-2404.

3. Polivy J, Herman CP. An evolutionary perspective on dieting. Appetite. 2006;47(1):30-35.

4. Sumithran P, Prendergast LA, Delbridge E, et al. Long-term persistence of hormonal adaptations to weight loss. $N$ Engl J Med. 2011; 365(17):1597-1604.

5. de Castro JM, Elmore DK. Subjective hunger relationships with meal patterns in the spontaneous feeding behavior of humans: evidence for a causal connection. Physiol Behav. 1988;43(2):159-165. 
6. Mela DJ. Eating for pleasure or just wanting to eat? Reconsidering sensory hedonic responses as a driver of obesity. Appetite. 2006; 47(1):10-17.

7. Herman CP, Roth DA, Polivy J. Effects of the presence of others on food intake: a normative interpretation. Psychol Bull. 2003; 129(6): 873-886.

8. Ceaser M. Hunger in primary anorexia nervosa. Am J Psychiatry. 1979; 136(7):979-980.

9. Slochower J. Emotional labeling and overeating in obese and normal weight individuals. Psychosom Med. 1976;38(2):131-139.

10. Slochower J, Kaplan SP, Mann L. The effects of life stress and weight on mood and eating. Appetite. 1981;2(2):115-125.

11. van Strien T. Ice-cream consumption, tendency toward overeating, and personality. Int J Eat Disord. 2000;28(4):460-464.

12. Harshaw C. Alimentary epigenetics: a developmental psychobiological systems view of the perception of hunger, thirst and satiety. Dev Rev. 2008;28(4):541-569.

13. Birch LL, Fisher JO, Davison KK. Learning to overeat: maternal use of restrictive feeding practices promotes girls' eating in the absence of hunger. Am J Clin Nutr. 2003;78(2):215-220.

14. Bruch H. Instinct and interpersonal experience. Compr Psychiatry. 1970;11(6):495-506.

15. Avalos LC, Tylka TL. Exploring a model of intuitive eating with college women. J Couns Psychol. 2006;53(4):486-497.

16. Satter E. The feeding relationship. J Am Diet Assoc. 1986;86(3):352-356.

17. Lovell-Smith D, Kenealy T, Buetow S. Eating when empty is good for your health. Med Hypotheses. 2010;75(2):172-178.

18. Cannon WB, Washburn AL. An explanation of hunger. Am J Physiol. $1912 ; 29: 441-454$.

19. Janssen P, Vanden Berghe P, Verschueren S, Lehmann A, Depoortere I, Tack J. The role of gastric motility in the control of food intake. Aliment Pharmacol Ther. 2011;33(8):880-894.

20. Sanger GJ, Hellstrom PM, Naslund E. The hungry stomach: physiology, disease, and drug development opportunities. Front Pharmacol. 2010;1:145.

21. Ciampolini M, Sifone M. Differences in maintenance of mean blood glucose (BG) and their association with response to "recognizing hunger." Int J Gen Med. 2011;4:403-412.

22. Le Magnen J. Hunger. Cambridge: Cambridge University Press; 1985.

23. Louis-Sylvestre J, Le Magnen J. Fall in blood glucose level precedes meal onset in free-feeding rats. Neurosci Biobehav Rev. 1980; 4(Suppl 1):13-15.

24. de Graaf C, Blom WA, Smeets PA, Stafleu A, Hendriks HF. Biomarkers of satiation and satiety. Am J Clin Nutr. 2004;79(6):946-961.

25. Melanson KJ, Greenberg AS, Ludwig DS, Saltzman E, Dallal GE, Roberts SB. Blood glucose and hormonal responses to small and large meals in healthy young and older women. J Gerontol A Biol Sci Med Sci. 1998;53(4):B299-B305.

26. Mayer J. Glucostatic mechanism of regulation of food intake. $N$ Engl J Med. 1953;249(1):13-16.

27. Lovell-Smith D. Diabetes: let the body speak its mind. $N$ Z Fam Physician. 1996;23(5):37-39.

28. Ciampolini M, Fognani G, van Weeren M, Borselli L. Attention to metabolic hunger for a steadier (SD decrease to $60 \%$ ), slightly lower glycemia $(10 \%)$ and body weight recovery in malnutrited infants [abstract]. Appetite. 2000;35:282.

29. Ciampolini M, Conti A, Bernardini S, Vicarelli D, Becherucci P, Seminara S. Internal stimuli controlled lower calorie intake: effects after eight months in toddler's diarrhoea. Ital J Gastroenterol. 1987;19: 201-204.

30. Ciampolini M, Vicarelli D, Seminara S. Normal energy intake range in children with chronic nonspecific diarrhea: association of relapses with the higher level. J Pediatr Gastroenterol Nutr. 1990;11(3): 342-350.

31. Ciampolini M. Requested meals versus scheduled meals. Int J Gen Med. 2012;5:345-353.

32. Ciampolini M, Bianchi R. Training to estimate blood glucose and to form associations with initial hunger. Nutr Metab (Lond). 2006;3:42.
33. Bernstein LM, Grossman MI. An experimental test of the glucostatic theory of regulation of food intake. J Clin Invest. 1956;35(6):627-633.

34. Levin BE. Glucosensing neurons do more than just sense glucose. Int J Obes Relat Metab Disord. 2001;25(Suppl 5):S68-S72.

35. Ciampolini M, Lovell-Smith D, Sifone M. Sustained self-regulation of energy intake. Loss of weight in overweight subjects. Maintenance of weight in normal-weight subjects. Nutr Metab (Lond). 2010;7:4.

36. Ciampolini M, Lovell-Smith H, Bianchi R, et al. Sustained selfregulation of energy intake: initial hunger improves insulin sensitivity. J Nutr Metab. 2010;2010:286952.

37. Wiesli P, Schaffler E, Seifert B, Schmid C, Donath MY. Islets secretory capacity determines glucose homoeostasis in the face of insulin resistance. Swiss Med Wkly. 2004;134(37-38):559-564.

38. Berridge KC. Motivation concepts in behavioral neuroscience. Physiol Behav. 2004;81(2):179-209.

39. Hill AJ, Blundell JE. Nutrients and behaviour: research strategies for the investigation of taste characteristics, food preferences, hunger sensations and eating patterns in man. J Psychiatr Res. 1982-1983; 17(2):203-212.

40. Hill AJ, Magson L, Blundell JE. Hunger and palatability: tracking ratings of subjective experience before, during and after the consumption of preferred and less preferred food. Appetite. 1984;5(4): 361-371.

41. Mattes RD, Friedman MI. Hunger. Dig Dis. 1993;11(2):65-77.

42. Tirosh A, Shai I, Tekes-Manova D, et al. Normal fasting plasma glucose levels and type 2 diabetes in young men. $N$ Engl J Med. 2005; 353(14):1454-1462.

43. Bastard JP, Maachi M, Lagathu C, et al. Recent advances in the relationship between obesity, inflammation, and insulin resistance. Eur Cytokine Netw. 2006;17(1):4-12.

44. Gustavsson CG, Agardh CD. Markers of inflammation in patients with coronary artery disease are also associated with glycosylated hemoglobin A1c within the normal range. Eur Heart J. 2004;25(23): 2120-2124.

45. Vozarova B, Weyer C, Lindsay RS, Pratley RE, Bogardus C, Tataranni PA. High white blood cell count is associated with a worsening of insulin sensitivity and predicts the development of type 2 diabetes. Diabetes. 2002;51(2):455-461.

46. Bloomgarden ZT. Insulin resistance: causes and consequences. Int Rev Neurobiol. 2005;65:1-24.

47. Biddinger SB, Kahn CR. From mice to men: insights into the insulin resistance syndromes. Annu Rev Physiol. 2006;68:123-158.

48. Ciampolini M, Borselli L, Giannellini V. Attention to metabolic hunger and its effects on Helicobacter pylori infection. Physiol Behav. 2000; 70(3-4):287-296.

49. Schvarcz E, Palmer M, Aman J, Horowitz M, Stridsberg M, Berne C. Physiological hyperglycemia slows gastric emptying in normal subjects and patients with insulin-dependent diabetes mellitus. Gastroenterology. 1997;113(1):60-66

50. Towne JB, Hamilton RF, Stephenson DV Jr. Mechanism of hyperalimentation in the suppression of upper gastrointestinal secretions. Am J Surg. 1973;126(6):714-716.

51. Barnett JL, Owyang C. Serum glucose concentration as a modulator of interdigestive gastric motility. Gastroenterology. 1988;94(3): 739-744.

52. Sachs G, Weeks DL, Melchers K, Scott DR. The gastric biology of Helicobacter pylori. Annu Rev Physiol. 2003;65:349-369.

53. Amieva MR, El-Omar EM. Host-bacterial interactions in Helicobacter pylori infection. Gastroenterology. 2008;134(1):306-323.

54. Ciampolini M, Bini S, Orsi A. Microflora persistence on duodenojejunal flat or normal mucosa in time after a meal in children. Physiol Behav. 1996;60(6):1551-1556.

55. Azpiroz F, Bouin M, Camilleri M, et al. Mechanisms of hypersensitivity in IBS and functional disorders. Neurogastroenterol Motil. 2007; 19(Suppl 1):62-88.

56. Hecht $\mathrm{G}$. In the beginning was Helicobacter pylori: roles for microbes in other intestinal disorders. Gastroenterology. 2007;132(2):481-483. 
57. Muller H, de Toledo FW, Resch KL. Fasting followed by vegetarian diet in patients with rheumatoid arthritis: a systematic review. Scand J Rheumatol. 2001;30(1):1-10.

58. Kjeldsen-Kragh J. Rheumatoid arthritis treated with vegetarian diets. Am J Clin Nutr. 1999;70(Suppl 3):594S-600S.

59. Stamp LK, James MJ, Cleland LG. Diet and rheumatoid arthritis: a review of the literature. Semin Arthritis Rheum. 2005;35(2) 77-94.

60. Lovell-Smith HD. Rheumatoid arthritis and Maharishi Ayur-Veda [letter]. N Z Med J. 1992;105(927):42.
61. Lovell-Smith HD. A Consideration of Homeostatic Regulation of Eating from the Perspective of Maharishi Vedic Science [doctoral thesis]. Auckland: The University of Auckland; 2009.

62. Cohen DA, Babey SH. Candy at the cash register - a risk factor for obesity and chronic disease. N Engl J Med. 2012;367(15):1381-1383.

63. Cook SA, Aitman T, Naoumova RP. Therapy insight: heart disease and the insulin-resistant patient. Nat Clin Pract Cardiovasc Med. 2005;2(5): 252-260. 


\section{Appendix \\ Glossary}

\begin{tabular}{|c|c|}
\hline Appetite & The desire to eat \\
\hline \multicolumn{2}{|l|}{ Blood glucose estimation } \\
\hline During training & $\begin{array}{l}\text { Writing the expected blood glucose value immediately before measuring the blood sample } \\
\text { by glucometer }\end{array}$ \\
\hline After training and validation & Evaluating one's own current blood glucose value without measurement \\
\hline Hunger Recognition & The meal pattern whereby initial hunger is recognized \\
\hline Initial hunger & $\begin{array}{l}\text { Low blood glucose-associated hunger: the empty hollow sensation and/or inanition at their } \\
\text { first appearance }\end{array}$ \\
\hline Initial hunger meal pattern & $\begin{array}{l}\text { A meal pattern in which initial hunger and low blood glucose is present pre-meal for most } \\
\text { meals }\end{array}$ \\
\hline Inanition & $\begin{array}{l}\text { Fatigue or light-headedness associated with lack of food: "A condition of exhaustion resulting } \\
\text { from lack of nourishment" (definition from: New Shorter Oxford English Dictionary, 1993) }\end{array}$ \\
\hline Migrating motor complex & A cycle of quiescence and contractions of the stomach and small intestine \\
\hline Phase III contractions & A series of high-amplitude contractions, which form part of the migrating motor complex \\
\hline
\end{tabular}

International Journal of General Medicine

Dovepress

\section{Publish your work in this journal}

The International Journal of General Medicine is an international, peer-reviewed open-access journal that focuses on general and interna medicine, pathogenesis, epidemiology, diagnosis, monitoring and treatment protocols. The journal is characterized by the rapid reporting of reviews, original research and clinical studies across all disease areas.

A key focus is the elucidation of disease processes and management protocols resulting in improved outcomes for the patient.The manuscript management system is completely online and includes a very quick and fair peer-review system. Visit http://www.dovepress.com/ testimonials.php to read real quotes from published authors.

Submit your manuscript here: http://www.dovepress.com/international-journal-of-general-medicine-journal 